\title{
Interação social e responsividade em ambientes doméstico e de creche: cultura e desenvolvimento
}

\author{
Eulina da Rocha Lordelo \\ Universidade Federal da Bahia
}

\begin{abstract}
Resumo
Visando descrever e comparar os níveis de interação em ambientes doméstico e de creche, incluindo diferentes indicadores de interação, o estudo observou 148 crianças em situação de brinquedo livre, 58 em creches e 90 em ambiente doméstico, de nível sócio econômico baixo e médio. As observações foram filmadas e segmentadas em 30 intervalos de 10 segundos, registrando-se as interações com adultos e outras crianças. Os resultados encontrados sugerem que casa e creche são diferentes quanto às oportunidades de interação, a casa favorecendo mais interações corporais, uma diferença não encontrada nas interações verbais. $\mathrm{O}$ conceito de interação, em sua relação com um quadro teórico de referência, emerge como crucial nesse estudo. Os resultados encontrados sugerem que os estudos na área problematizem os critérios de interação adotados, no sentido de buscar uma descrição mais abrangente do ambiente de desenvolvimento, em que o papel da cultura seja equacionado.
\end{abstract}

Palavras chave: Interação social, Responsividade, Ambiente de desenvolvimento.

\begin{abstract}
Social interaction and responsiveness in home and day-care centres environments: Culture and development. Aiming to describe and compare the interactional levels in home and day-care centres environments, including different indicators of interaction, this study observed 148 children in free play situations, 58 in day-care centres and 90 in home environment, from low and medium SES. The observations were filmed and segmented in 30 intervals of 10 seconds. All adult-child and child-child interactions were coded. The results found suggest that home and day-care centres are different about the opportunity of interactions; at home, there are more body interactions, but this difference is not found in verbal interactions. The interaction concept seems crucial at this study. The results suggest that studies in the area should discuss the interaction criteria used, aiming a more comprehensive description about the development environment, in which the role of culture may be considered included.
\end{abstract}

Key words: Interaction, Responsiveness, Development environment.

A avaliação da qualidade do ambiente de desenvolvimento da criança é um tópico de investigação relevante para as políticas públicas de atenção à infância. O tema esbarra, entretanto, nas dificuldades de definição: o que é exatamente um bom ambiente de desenvolvimento (Baumrind, 1993; Scarr, 1993; Scarr \& Eisenberg, 1993; Smith, 1976).

À parte certos atributos universalmente reconhecidos como essenciais para o desenvolvimento, como a disponibilidade de cuidados físicos básicos (alimentação, segurança física, cuidados médicos), o campo é pontuado por disputas teóricas e mesmo ideológicas (Jackson, 1993). A partir da emergência da teoria do apego e da descoberta dos mecanismos subjacentes à formação dos vínculos (responsividade materna), derivou-se um conceito de ambiente de desenvolvimento em que as relações da criança com um adulto sensível, capaz de reconhecer os sinais da criança e responder a eles apropriadamente, constituiriam o modo mais favorável para o desenvolvimento (Bowlby, 1984).

Os alegados riscos da creche como ambiente de desenvolvimento, as idéias de privação cultural e os mecanismos criados para reduzi-la, bem como diversos estudos relatando efeitos prejudiciais de certos contextos de criação (famílias monoparentais, pais divorciados etc) vêm sendo intensamente discutidos, com base no questionamento sobre a tradição de pesquisa que lhe serve de fundamento, vista como cultural e ideologicamente enviesada, como é o caso da pesquisa sobre apego. 
A avaliação e a comparação de creches como ambientes de desenvolvimento inserem-se neste campo de debates. Questiona-se a adequação da creche como ambiente de desenvolvimento em vista de seus possíveis riscos para a criança, determinados pela sua incapacidade em prover o tipo de cuidado requerido para a criança, especialmente nos primeiros anos. Os estudos que dão suporte a essas preocupações têm se dedicado a avaliar a qualidade do ambiente, considerando os diversos contextos de desenvolvimento usuais na maior parte do mundo ocidental industrializado, incluindo o nosso país, enfatizando fortemente indicadores de responsividade do adulto. $\mathrm{O}$ conceito de responsividade vem se tornando um instrumento crescentemente importante na descrição e explicação de resultados desenvolvimentais, uma vez que ele está relacionado à formação dos vínculos de apego (Isabella \& Belsky, 1991) e ao desenvolvimento cognitivo (Papousek \& Papousek, 1984; Schaffer, 1992). Entretanto, os indicadores de responsividade empregados nas pesquisas são variados, justificando a preocupação com sua definição como uma das bases para apreciar os resultados das pesquisas.

Lordelo, Fonseca e Araújo (2000) estudaram 45 pares de díades mãe filhos em ambiente doméstico e, através de indicadores construídos sobre observação de suas interações, classificaram as mães quanto ao nível de responsividade, encontrando entre as mães de nível sócio-econômico médio escores mais de duas vezes mais altos do que o observado em mães de nível sócio-econômico baixo. Os indicadores foram selecionados com base na literatura sobre responsividade e nos itens da escala de avaliação HOME (Caldwell \& Bradley, 1978) que apareciam nas situações de teste. Diferenças tão altas em responsividade, supondo-se que esta esteja associada a diferentes resultados desenvolvimentais, são preocupantes e conduzem a dois tipos de questionamento. $\mathrm{O}$ primeiro é a possibilidade de que as medidas empregadas sejam contaminadas por algum tipo de viés cultural e, segundo, haveria alguma teoria capaz de explicar satisfatoriamente essas diferenças?

Uma descrição impressionista sobre os ambientes em que as díades foram observadas pode contribuir para ilustrar a dúvida: os estilos de cuidado, os ambientes físicos, a atitude geral da mãe em relação à criança, eram muito diferentes, de fato. Enquanto as mães de classe média parecem organizar suas rotinas a serviço da criança, as mães de classe baixa aparentemente "encaixam" a criança nas suas atividades, um comportamento muito adaptativo numa situação em que não há outros cuidadores. À parte as diferenças individuais no estilo de cuidado, pode-se identificar uma configuração relacionada ao contexto, uma marca do sistema de crenças e práticas, diferenciável conforme o nível sócio-econômico. Essas características são passíveis de apreensão pelos instrumentos de avaliação da responsividade?

Por exemplo, De Wolff e van Ijzendoorn (1997) realizaram uma meta-análise de 66 estudos sobre a relação entre sensibilidade maternal e segurança do apego. Eles encontraram 55 diferentes constructos que se referiam a vários aspectos do comportamento maternal, sendo a freqüência de seu uso bastante variável; enquanto um indicador como Sensibilidade foi usado em 30 estudos e Suporte em 21, um indicador como Contato físico esteve presente em apenas nove.

Além disso, a investigação da responsividade tende a descrevê-la como traço de personalidade materna, provavelmente subestimando o peso das variáveis culturais associadas às práticas de cuidado. Por exemplo, Isabella e Belsky (1991) avaliaram o nível de sincronia entre díades mãe filho, usando 12 categorias de respostas maternas, a maioria delas relacionada à interação exclusiva entre adulto e criança, a maior parte verbal, excluindo o simples contato físico. Essas categorias parecem totalmente apropriadas para o contexto de criação contemporâneo das sociedades industriais, entre as classes médias (família nuclear, nível de instrução médio a alto, poucos filhos e cuidado individual à criança), mas, provavelmente, ajustam-se pouco a outros contextos de criação (famílias de nível sócio-econômico baixo, baixa instrução, maior número de filhos e, principalmente, diferentes concepções e valores de criação). Interessantemente, a meta-análise empreendida por De Wolff e van Ijzendoorn (1997) sobre a relação entre sensibilidade maternal e segurança do apego identificou um decréscimo de confiabilidade dos resultados quando os estudos foram segmentados por classe social; quando as amostras eram provenientes de extratos sócioeconômicos baixos, a relação encontrada entre responsividade e apego foi mais baixa e menos consistente.

Seria, então, possível que o conceito de responsividade seja subordinado a um tipo de viés contextual/cultural? Como descrever e comparar diferentes ambientes de desenvolvimento quanto ao nível de responsividade? Parece necessária uma problematização do conceito e suas implicações em termos metodológicos. Ao mesmo tempo, subsiste a pergunta central da Psicologia do desenvolvimento: responder como os organismos se desenvolvem implica em explicar as continuidades (ou descontinuidades) que ligam as diversas etapas do caminho que os organismos percorrem ao longo da sua vida. Desse modo, os diferentes modelos de desenvolvimento têm a tarefa de descrever e explicar as transações entre organismos e ambientes e como elas estão relacionadas às mudanças que chamamos o ciclo de vida.

De fato, o que está em jogo é a relação entre ambiente e comportamento, uma relação central a diversas abordagens em Psicologia. O modo pelo qual ambiente é concebido, entretanto, varia enormemente, seguindo o conjunto de pressupostos teóricos e metodológicos que caracterizam o campo. Os mais recentes desenvolvimentos da Psicologia ambiental têm enfatizado os efeitos das características físicas ou sociais do ambiente, como percebidas pelo sujeito. Verifica-se um acentuado desenvolvimento e sofisticação de abordagens ao assunto, como se observa na teoria ecológica de Bronfenbrenner (1979/1996; Bronfenbrenner \& Ceci, 1994, Bronfenbrenner, Moen, Elder \& Luscher, 1995) que abrange, sob o conceito de ambiente, as dimensões mais próximas e estritamente físicas do constructo ambiente até aquelas mais intangíveis como cultura, valores e ideologias, incorporando aos modelos de descrição e explicação do desenvolvimento os elementos pessoa, processo, contexto e tempo. A teoria ecológica tem influenciado grande número de estudos em 
desenvolvimento humano (Alves \& cols., 2001; Tudge, Doucet \& Odero, 1999; Tudge et al., 2000), pelo seu potencial de rastrear os efeitos construtivos que ambiente e organismo exercem um sobre o outro.

Apesar desses avanços em propor quadros de referência holísticos, capazes de abarcar mais aspectos do comportamento, permanece uma lacuna em explicar os mecanismos pelos quais organismo e ambiente influenciam-se mutuamente. Por exemplo, sabe-se que os indivíduos regulam seus comportamentos e ambientes para otimizar as características mais favoráveis ao seu desenvolvimento (Lordelo, 1995). De certa forma, os organismos se desenvolvem como sistemas abertos, em que os resultados finais não podem ser inferidos das variáveis iniciais (Thelen, 1989; Valsiner, 1987).

Naturalmente, essa imprevisibilidade não significa que o desenvolvimento é randômico. Mas ainda resta identificar, nas palavras de Keller (1999), um laço entre cultura e biologia, que explique o desenvolvimento humano como um empreendimento conjunto entre organismos e ambientes relativamente estruturados isoladamente, mas com propriedades emergentes resultantes da sua interação.

O modo como base biológica e a cultura interagem para formar indivíduos diferenciados deve ser estudado nos ciclos de vida e é o objeto da Psicologia evolucionária, que se propõe a fornecer respostas para as mais importantes preocupações desenvolvimentais, a saber: as interfaces entre biologia e cultura, as questões da plasticidade e continuidade e a sequiência das tarefas desenvolvimentais (Keller, 1996).

$\mathrm{Na}$ teoria do investimento parental, os esforços reprodutivos dos indivíduos estão relacionados com seus esforços somáticos. Embora a espécie humana seja, por excelência, uma espécie de alto investimento parental, diferenças quantitativas conduzem a dois estilos prototípicos de trajetórias desenvolvimentais: o Tipo 1 caracteriza o indivíduo que na infância experimenta um ambiente de escassez de recursos, com baixo investimento parental e clima familiar insatisfatório; o Tipo 2 descreve o indivíduo que, vivendo uma situação de afluência, experimenta um alto investimento parental e um clima familiar positivo. Ao lado de conseqüências psicológicas nas relações de apego entre criança e pais, os dois tipos de experiências levariam a diferenças no timing da puberdade, com o Tipo 1 amadurecendo bem mais cedo que o Tipo 2. Essas trajetórias continuariam a se diferenciar nos estilos reprodutivos subseqüentes, sendo o Tipo 1 mais quantitativo - mais filhos, menor espaçamento entre os nascimentos e baixo investimento parental e o Tipo 2 mais qualitativo - menos e mais espaçados filhos e alto investimento parental (Keller, 1996; Moffit, Caspi, Belsky \& Silva, 1992).

$\mathrm{O}$ investimento parental é definido geralmente em termos quantitativos, referindo-se à maior ou menor quantidade de cuidado biológico e/ou psicológico dispensado à prole. As decisões de investimento parental são baseadas nas condições ecológicas que os pais vivenciam e que se expressam na prevalência de diferenciados sistemas de cuidado. Essas estratégias são, obviamente, inconscientes.

Segundo Keller (1998), a primeira fase do desenvolvimento humano, denominada fase de aquisição, é caracteriza- da pela tarefa essencial de aquisição de uma matriz social primária, que deve ser adaptativa com respeito ao ambiente em que se desenvolve. A autora propõe que todos os contextos de desenvolvimento podem ser classificados segundo as dimensões do ambiente social e da estrutura de atenção (se exclusiva ou compartilhada com outros objetos). $\mathrm{O}$ investimento parental, expresso em sistemas diferenciados de cuidado, deve ter evoluído ao longo da história filogenética da espécie, para responder a problemas de adaptação postos pela seleção natural. A variedade de condições ecológicas encontradas no mundo atual, entretanto, deve exercer pressões para o uso de diferentes estratégias reprodutivas, incluindo o investimento parental, de modo a clarificar melhor a idéia de responsividade materna, tida pelos teóricos do apego como uma resposta parental universal.

A proposta da autora supõe a existência de quatro sistemas de cuidado parental: cuidado primário, contato corporal, sistema de estimulação e sistema face a face, os quais responderiam a diferentes necessidades da criança, mais ou menos freqüentes e associados funcionalmente aos contextos ecológicos nos quais estão inseridos. A prevalência de um ou outro desses sistemas estaria associada também a diferentes resultados desenvolvimentais.

Essa teoria supõe que os estilos reprodutivos dos indivíduos são principalmente não intencionais, não conscientes e implícitos, sendo regulados por uma Psicologia complexa capaz de processar e integrar informação, motivações, desejos, crenças, atitudes e comportamentos referentes ao sucesso reprodutivo (Keller, 1996).

Poderia uma teoria do investimento parental explicar os diferentes estilos de cuidado à criança, observados em diferentes contextos sócio-econômicos? Keller (1998) considera que existem dois padrões de experiências iniciais de socialização diferenciados nas culturas: ocidental, estruturado na atenção exclusiva sob a forma de interações diádicas; e nãoocidental, caracterizado pela atenção co-ativa, como interações sociais acontecendo com múltiplos cuidadores.

Segundo Rabinovich (1998), a sociedade brasileira seria formada por uma mistura de culturas de origem indígena, africana e européia, o que favoreceria a permanência de traços de um sistema cultural não-ocidental, característico de certas camadas da população, especialmente as mais pobres.

Assim, o estudo da responsividade dos ambientes de desenvolvimento deve adotar estratégias de investigação apropriadas aos diferentes contextos culturais em que ocorrem. Essas diferenças poderiam ser críticas para a introdução de um viés na pesquisa: como os pesquisadores são educados segundo padrões da cultura ocidental, eles tenderiam a avaliar as práticas observadas sem referência ao sistema cultural em que estão inseridas.

Assim, justificam-se estudos que contribuam com descrições mais abrangentes, levando em conta as eventuais especificidades culturais características dos grupos investigados. Portanto, o presente trabalho propôs-se a descrever e comparar indicadores de responsividade em ambientes doméstico e de creche nível característicos de dois ambientes sócio econômicos diferentes. $\mathrm{O}$ estudo insere-se no contexto 
de um projeto de investigação maior, sobre a responsividade dos ambientes de desenvolvimento contemporâneos.

Especificamente, pretendeu-se realizar um exercício teórico-metodológico, através do uso de diferentes indicadores de responsividade relacionados com o nível de interação entre adulto e criança: dependendo dos critérios de interação adotados, os resultados dos níveis de interação seriam modificados? Esses diferentes critérios podem ser sustentados (fundamentados) teoricamente?

\section{Método}

\section{Sujeitos}

O estudo observou 148 crianças em situação de brinquedo livre. Desse total, 58 pertenciam a quatro creches diferentes, divididas em dois grupos, um de creches públicas (36 crianças) e outro de creches particulares (22 crianças), com idade variando entre 13 a 35 meses. No ambiente doméstico, foram observadas 90 crianças residentes em diferentes bairros, compondo dois grupos, um de classe média (45 crianças) e outro de classe baixa (45 crianças), com idades entre 12 e 42 meses. Do total da amostra, 70 crianças eram do sexo masculino e 78 eram do sexo feminino. A designação de creche pública ou privada, neste caso, correspondeu a nível sócio econômico médio ou baixo, sendo essa classificação resultante de inferências acerca da renda, escolaridade e ocupação dos pais, dados fornecidos pelas creches ou diretamente pelas famílias para as crianças observadas em ambiente doméstico.

\section{Coleta de dados}

No ambiente doméstico, filmou-se a díade mãe-criança enquanto a mãe respondia a questões gerais relativas à maternidade e às práticas de cuidado à criança. A criança permaneceu no local da entrevista, possibilitando a observação e o registro das interações da díade. Planejou-se a observação como uma situação de demandas conflitantes, de modo a aproximar, o mais possível, o ambiente doméstico das condições da creche. Essa decisão foi tomada para reduzir a reco- nhecida desvantagem da creche em termos da atenção do adulto, de forma a tornar a comparação mais cautelosa, reduzindo um possível efeito de viés, uma vez que existia uma expectativa de déficit de responsividade adulta na creche.

$\mathrm{Na}$ situação de creche, os procedimentos de filmagem incluíram três sessões de 10 minutos de duração, realizadas em dias diferentes num intervalo de tempo inferior a 15 dias. As educadoras receberam instruções para agir naturalmente, interagindo como de costume. A filmagem acompanhou uma criança por vez, procurando incluir os alvos da atenção da criança, tanto objetos como pessoas, outras crianças ou adultos.

\section{Tratamento e análise dos dados}

$\mathrm{O}$ procedimento de tratamento dos dados segmentou as observações em 30 intervalos de 10 segundos. Caracterizouse a interação a partir de um critério mínimo definidor, a orientação mútua - um parceiro dirige-se ao outro o qual, por sua vez, demonstra perceber a iniciativa - com duração de, no mínimo, três segundos. Essa definição incluiu situações em que o adulto segura a criança no colo, ainda que a criança esteja voltada de costas para ele. Categorizou-se cada intervalo segundo quatro classes de interação: a) Sozinho, b) Interação com adulto e outra criança, c) Interação criançacrianca e d) Interação criança-adulto. As categorias de Interação criança-criança e Interação criança-adulto foram divididas em subcategorias de acordo com o critério de incluírem ou não comunicação verbal. As médias das categorias foram cruzadas com sexo e idade da criança, tipo de creche (pública ou privada) e com o nível sócio-econômico das famílias dos ambientes domésticos, sendo as comparações testadas por ANOVA (análise de variância).

\section{Resultados}

\section{Interações em ambiente doméstico}

Como se pode ver na Figura 1, a média das interações não verbais com adultos (todas as formas de contato que

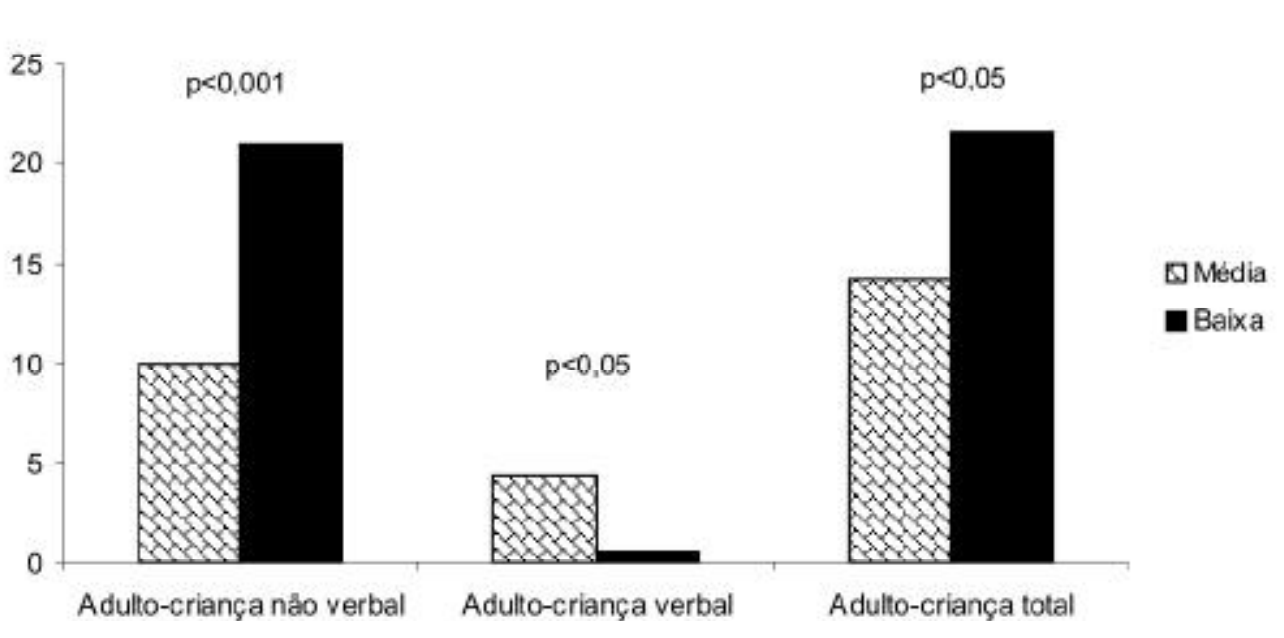

Figura 1

Média das interações verbais e não verbais adulto e criança por situação sócio-econômica, em ambiente doméstico. 


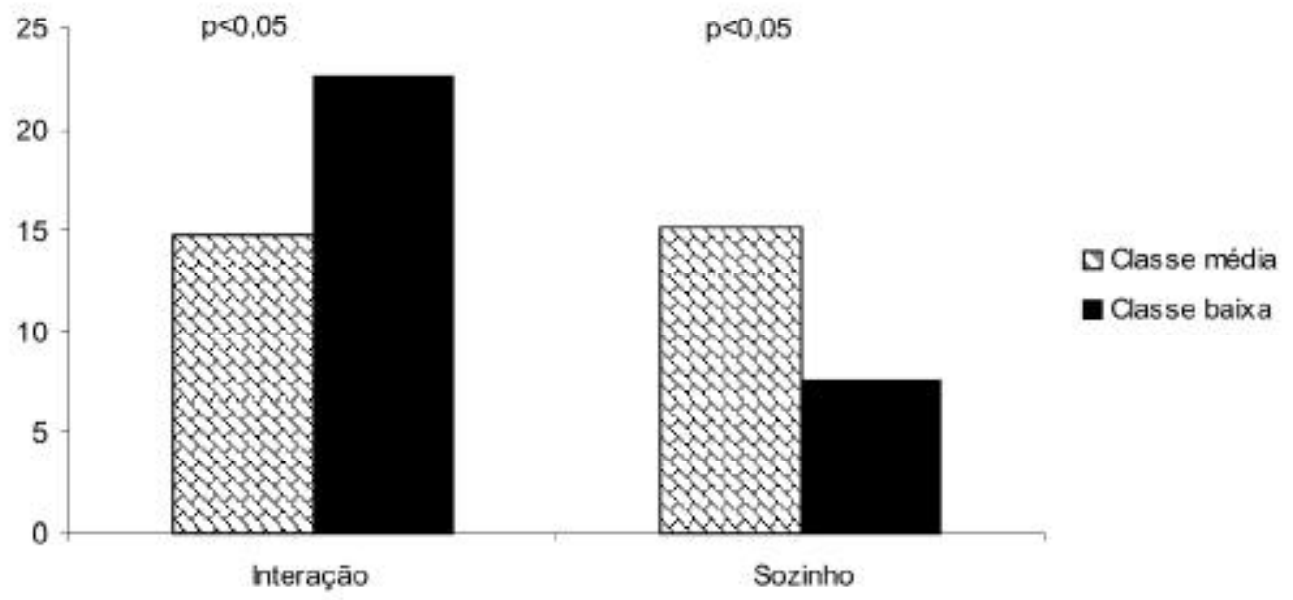

Figura 2

Média dos episódios em que a criança passa interagindo e os que ela está sozinha por situação sócio econômica, em ambiente doméstico.

envolvessem pelo menos orientação mútua) foi significativamente maior no nível sócio econômico baixo, assim como as interações gerais entre adulto e criança. Mas quando a interação é acompanhada de comunicação verbal com um adulto, verifica-se que as mães com nível sócio econômico mais elevado atingem médias mais elevadas. Somando-se todas as interações (verbais e apenas corporais), as díades de nível sócio econômico baixo ainda apresentaram médias mais altas, o que representa claramente o maior peso das interações apenas corporais no conjunto.

As crianças de classe média passam mais tempo sozinhas e apresentam menos episódios de interação do que as crianças de classe baixa. As mães das famílias de nível sócio econômico médio tendem a deixar seus filhos mais "soltos" e independentes para explorar o ambiente por conta própria, ao contrário das mães de classe baixa, que seguram suas crian- ças no colo por longos períodos durante a realização da entrevista.

Como pode ser visto na Figura 3, as interações entre as díades de nível sócio-econômico baixo são muito mais freqüentes nas interações não verbais, que envolvem só o contato corporal, enquanto as díades de nível sócio econômico médio obtêm resultados mais altos nas interações verbais.

\section{Interações em ambiente de creche}

As crianças das creches públicas apresentam um número de episódios de interação não verbal significativamente maior $(\mathrm{p}<0,05)$ do que as crianças de creche particular, como se pode observar na Figura 4. Já em relação à interação verbal, não há diferenças significativas na frequiência dos episódios.

As díades estudadas no contexto doméstico apresentam um número de episódios de interação não verbal significati-

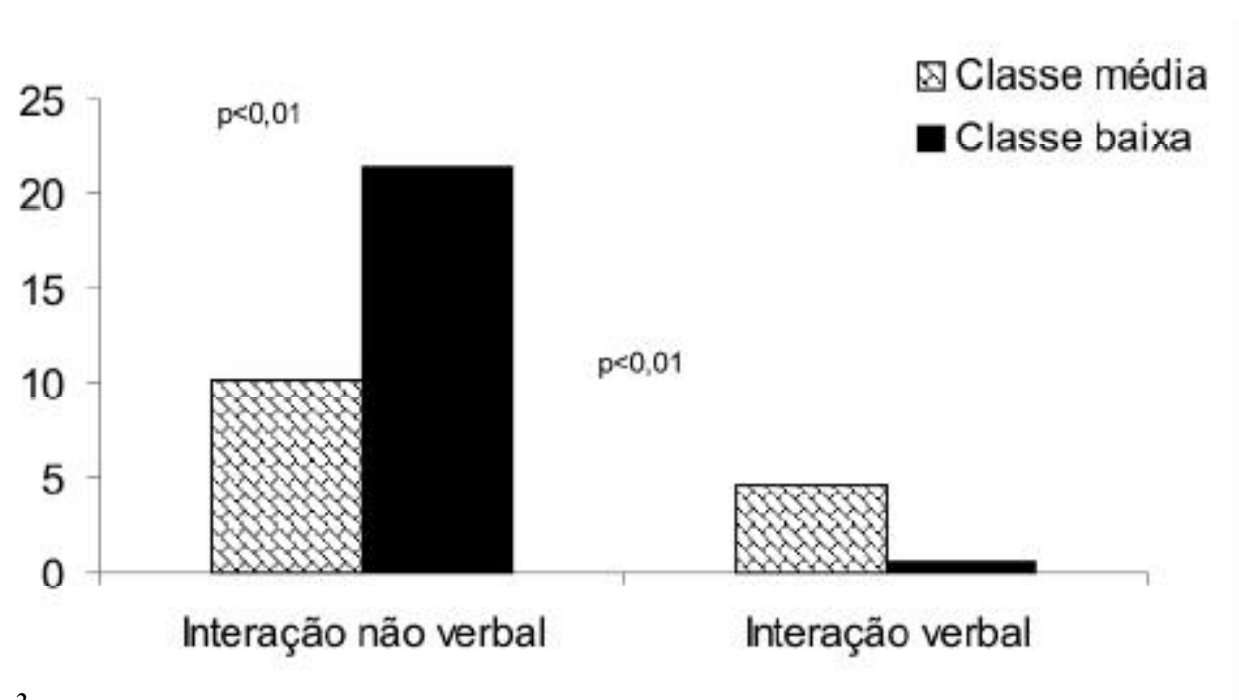

Figura 3

Média das interações não verbais e verbais por situação sócio econômica, em ambiente doméstico. 


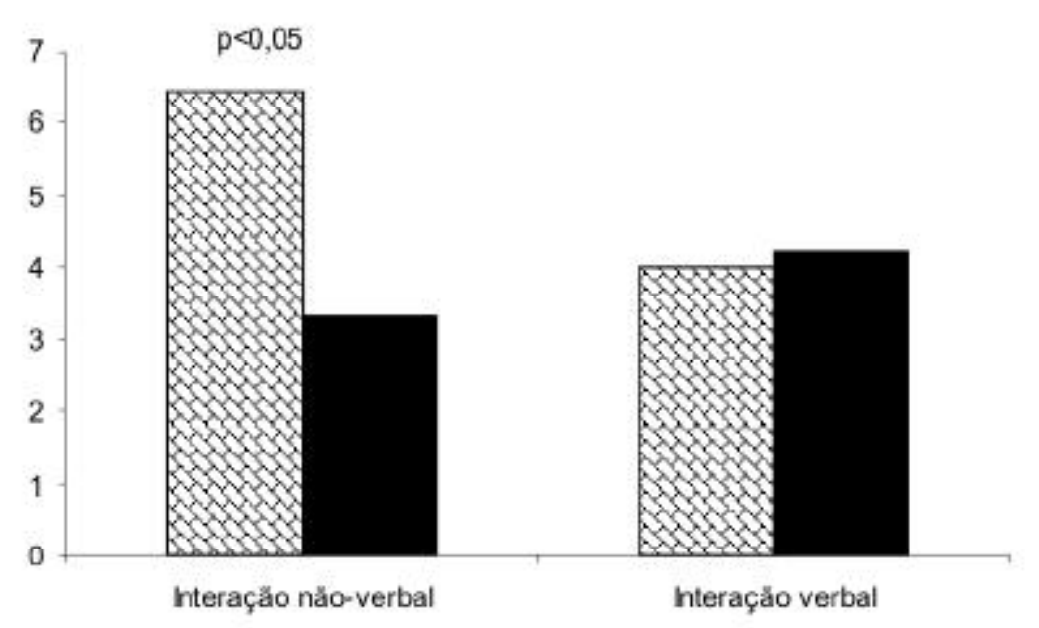

$\checkmark$ Creche pública

- Creche particular

Figura 4

Média das interações verbais e não verbais por tipo de creche.

vamente maior $(\mathrm{p}<0,001)$ do que aquelas no contexto de creche, como pode ser visto na Figura 5. Em relação à interação verbal, não há diferenças significativas.

\section{Discussão e Conclusões}

Os resultados encontrados fortalecem os achados de outros estudos da literatura quanto à influência do tipo de ambiente na interação. Certas configurações de ambiente são mais favoráveis à interação em geral e à interação entre certos parceiros (Campos de Carvalho, 1998; Padovani \& Campos de Carvalho, 1997). A creche e casa são diferentes quanto às oportunidades de interação, a casa favorecendo mais interações corporais, uma diferença não encontrada nas interações verbais. No geral, pode-se dizer que creches públicas e casas de nível sócio-econômico baixo assemelhamse quanto ao contato corporal, superior ao encontrado no nível sócio-econômico médio e nas creches privadas; essa relação, entretanto, não pode ser feita quanto ao nível de interação verbal: esta é diferente na comparação entre ambientes domésticos (mais alta na classe média) e semelhante nos dois tipos de creche.

A compreensão desses resultados e de suas implicações requer, preliminarmente, a discussão do conceito de interação. Os resultados mostram-se bastante diferentes dependendo dos critérios empregados para definir interação. Se a definição de interação se restringir às comunicações verbais, em que adulto e criança conversam voltados um para o outro, as condições do ambiente doméstico de classe baixa teriam que ser consideradas muito inferiores às encontradas nos grupos de classe média. Ao contrário, se a interação incluir obrigatoriamente o contato físico, seriam talvez os ambientes de classe média a serem considerados deficientes. Assim, parece necessário especificar os diferentes níveis em que o sistema de cuidado pode ser exercido, variando do simples cuidado primário até formas de interação mais complexas (Keller, 1998), as quais podem ter repercussões diferentes de acordo com a

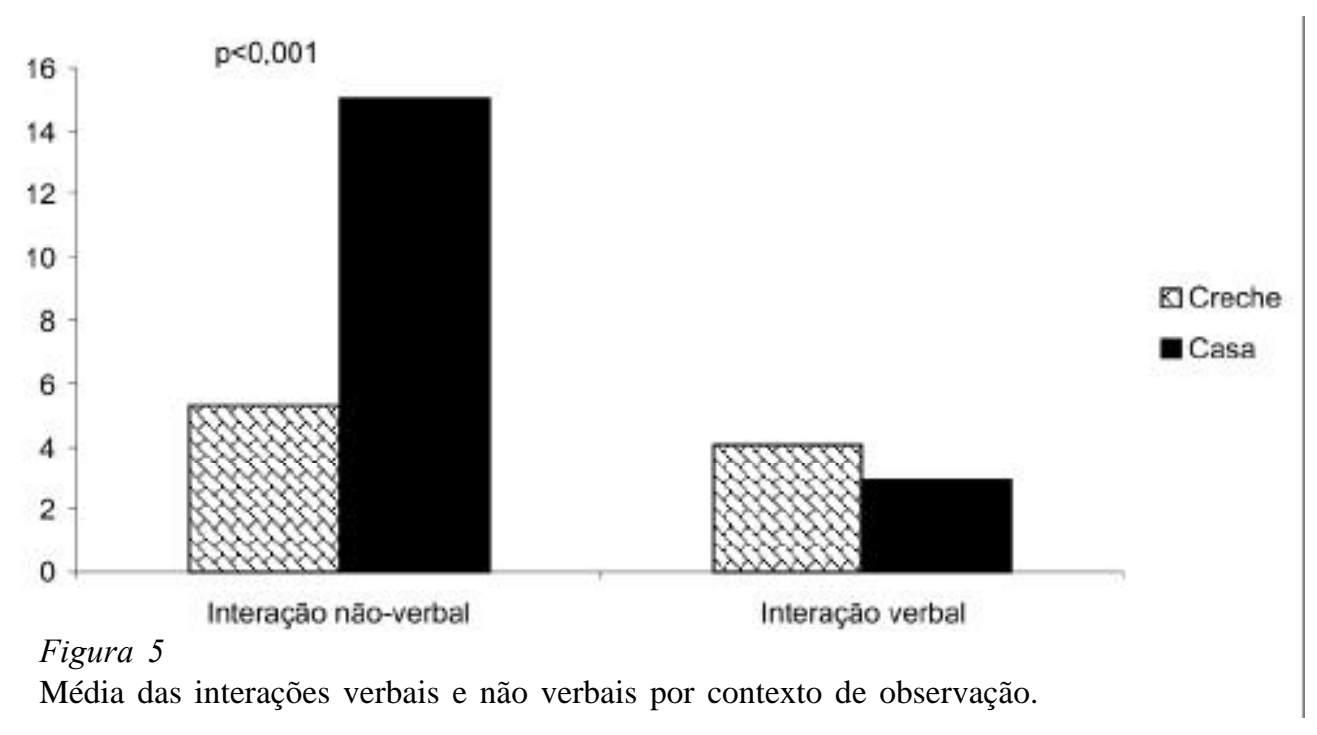


esfera do desenvolvimento em questão, como personalidade, estilos cognitivos, competências sociais, entre outras em diferentes áreas do desenvolvimento.

Desse modo, evidencia-se a importância de ajustar decisões metodológicas aos quadros teóricos de referência que lhe dão suporte. No estudo dos ambientes de desenvolvimento, a adoção de um conceito de responsividade deve levar em conta a visão do desenvolvimento como empreendimento biológico, com sua lógica de aptidão, de um lado, e de outro, como empreendimento sócio cultural, com sua lógica própria, orientada por objetivos sociais característicos de uma época, classe social, cultura. Embora essas duas lógicas interliguem-se freqüentemente, pode acontecer que a pesquisa científica tome uma pela outra, com consequiências prejudiciais à compreensão dos fenômenos do desenvolvimento humano.

Assim, tomar o sistema de cuidado face a face (nos termos de Keller, 1998) como representativo de um alto grau de responsividade do ambiente leva o pesquisador a concluir que os ambientes de crianças de nível sócio econômico baixo não são responsivos. O que este estudo pretendeu sugerir é que isso pode ser verdadeiro apenas para este nível de sistema de cuidado mais elevado, compatível com uma certa configuração ecológica em que os recursos físicos e a cultura local permitem um alto nível de investimento parental. Em outras circunstâncias, por exemplo, em ambientes pobres em recursos materiais, um ambiente de desenvolvimento baseado fortemente no sistema de cuidado face a face seria talvez pouco adaptativo, resultando em prejuízo para a família e, por extensão, para a própria criança.

Além disso, o presente estudo contribui para deslocar a atenção dos pesquisadores para o fenômeno da responsividade materna enquanto traço de personalidade, em direção a um campo em que as práticas observadas estão conectadas a sistemas culturais originados e sustentados por circunstâncias ecológicas particulares. Esse deslocamento não pretende reduzir o papel de tais fatores - além de traços de personalidade, circunstâncias relativas aos diversos níveis do ambiente (micro, meso, exossistemas e macrossistemas), como o suporte disponível para a criação dos filhos, na forma de apoio familiar, comunitários e dos sistemas públicos de assistência, bem como fatores pessoais passageiros como estado de saúde, tudo isso pode afetar as práticas de cuidado parentais e a construção do vínculo com a criança (Bronfenbrenner, 1979/1996; Bronfenbrenner \& Ceci, 1994, Bronfenbrenner, Moen, Elder \& Luscher, 1995). Em vez disso, o estudo pretende enfatizar a necessidade de considerar as diversas fontes de influência capazes de explicar as variações encontradas em responsividade e nos seus efeitos sobre a formação dos vínculos de apego.

Os resultados encontrados sugerem, portanto, que os estudos na área, além de explicitarem suas definições de interação, como é usualmente feito, problematizem os critérios adotados, no sentido de buscar uma compreensão mais abrangente do fenômeno. Tal compreensão pode ser importante para superar as avaliações dos ambientes de desenvol- vimento como bons ou maus, alcançando-se uma compreensão de efeitos particulares e substantivos relacionados às diversas configurações. Por exemplo, Keller (1999) concorda em que as práticas de cuidado à criança têm um impacto direto na formação da personalidade, uma idéia antiga já presente em diversos autores como Mead (1968) e Whiting (1983) e procura identificar os mecanismos através dos quais essas diferenças são criadas, relacionando-as às propensões biológicas da espécie, ajustadas pelas pressões de variáveis ecológicas, expressas no investimento parental.

Essas perspectivas podem ser significativas para libertar a Psicologia do desenvolvimento do viés do etnocentrismo do pesquisador, ao definir e diferenciar resultados desenvolvimentais socialmente desejados e resultados desenvolvimentais biologicamente adaptados (Scarr, 1993; Smith, 1976; Weisner \& Gallimore, 1977).

Essa diferenciação não implica, naturalmente, em um menor compromisso com políticas públicas capazes de melhorar o bem estar humano, especialmente da criança, mas, tão somente em ampliar o poder explicativo das teorias sobre o desenvolvimento. Nossa cultura - ocidental, urbana e pós industrial, atribui um alto valor às habilidades acadêmicas, centradas na dimensão cognitiva do desenvolvimento e a serviço, geralmente, de objetivos de vida ligados à acumulação, ao consumo de bens e à realização pessoal. Da mesma forma, dimensões de personalidade como iniciativa e independência são tipicamente valorizadas em culturas com um forte senso de prioridade do indivíduo. Nesse tipo de contexto, é possível que sistemas de cuidado apropriados para gerar resultados compatíveis com os valores sociais vigentes devam ser privilegiados enquanto políticas públicas, não devendo esses resultados, entretanto, ser tomados como equivalentes ao desenvolvimento humano, enquanto conceito.

\section{Agradecimentos}

A autora registra agradecimentos pelo apoio do CNPq/ PRPPG/PIBIC-UFBa.

\section{Referências}

Alves, P. B., Koller, S. H., Silva, A. S., Reppold, C. T., Santos, C. L., Bichinho, G. S., Prade, L. T., Silva, M. R., \& Tudge, J. (1999). A construção de uma metodologia observacional para o estudo de crianças em situação de rua: criando um manual de codificação de atividades cotidianas. Estudos de Psicologia - Natal, 4(2), 289-310.

Baumrind, D. (1993). The average expectable environment is not good enough: a response to Scarr. Child Development, 64, 1299-1317.

Bowlby, J. (1984). Apego. São Paulo: Martins Fontes.

Bronfenbrenner, U., \& Ceci, S. J. (1994). Nature-nurture reconceptualized in developmental perspective: a bioecological model. Psychological Review, 101(4), 568-586.

Bronfenbrenner, U. (1979/1996). A ecologia do desenvolvimento humano: experimentos naturais e planejados. Porto Alegre: Artes Médicas.

Bronfenbrenner, U., Moen, P., Elder Jr, G. H., \& Luscher, K. (1995). Developmental ecology through space and time: A future perspective. In P. Moen, G.H. Elder Jr \& K. Luscher (Orgs.), Examining lives in context Perspectives on the ecology of human development (pp. 619-648). Washington: American Psychological Association. 
Caldwell, B. M., \& Bradley, R. H. (1978). Administration manual: Home observation on measurement of the environment (Manual). Litlle Rock: University of Arkansas.

Campos de Carvalho, M. I. (1998). Organização do espaço em creches e préescolas [Resumo]. Em Sociedade Brasileira para o Progresso Científico (Org.), Resumos - 50 Reunião Anual da SBPC. Natal: Autor.

De Wolff, M. S., \& van Ijzendoorn, M. H. (1997). Sensitivity and attachment: a meta-analysis on parental antecedents of infant attachment. Child Development, 68, 571-591.

Isabella, R. A., \& Belsky, J. (1991). Interactional synchrony and the origins of infant-mother attachment: a replication study. Child Development, 62, 373-384.

Jackson, J. F. (1993). Human behavioral genetics, Scarr's theory and her views on interventions: a critical review and commentary on their implications for african-american children. Child Development, 64, 1318-1333.

Keller, H. (1996). Abordagens evolucionárias. In J. W. Berry, Y. H. Pootinga \& J. Pandey (Orgs.), Handbook of cross-cultural psychology (vol 1, pp. 215256). Boston: Allyn and Bacon.

Keller, H. (1998). Diferentes caminhos de socialização até a adolescência. Revista Brasileira de Crescimento e Desenvolvimento Humano, 8(1/2), 01-14.

Keller, H. (1999, maio). Developmental pathways to individualism and interelatedness. Conferência apresentada na abertura do I Congresso NorteNordeste de Psicologia. Universidade Federal da Bahia/Conselho Regional de Psicologia - Terceira Região. Salvador, Bahia.

Lordelo, E. R. (1995). Ambiente de desenvolvimento humano - uma reflexão a partir do contexto creche. Tese de doutorado não-publicada. Universidade de São Paulo, São Paulo.

Lordelo, E. R., Fonseca, A. L., \& Araújo, M. L. V. B (2000). Responsividade do ambiente de desenvolvimento: crenças e práticas como sistema cultural de criação de filhos. Psicologia: Reflexão e Crítica, 13(1), 73-80.

Mead, M. (1968). Growing up in New Guinea. New York: Dell.

Moffit, T. E., Caspi, A., Belsky, J., \& Silva, P. A. (1992). Child Development, 63(1), 47-58.

Padovani, F., \& Campos-de-Carvalho, M.I. (1997). Arranjos espaciais preferenciais e ocasionais entre crianças pequenas em creche [Resumo]. In Sociedade
Interamericana de Psicologia (Org.), XXVI Congresso Interamericano de Psicologia-Resumos (p. 329). São Paulo: Autor.

Papousek, H., \& Papousek, M. (1984). Learning and cognition in the everyday life of human infants. In J. S. Rosenblatt (Org.), Advances in the study of behavior (vol. 14, pp.127-159). New York: Academic Press.

Rabinovich, E. (1998). Modos de morar no Brasil e contexto de desenvolvimento [Resumo]. In Sociedade Brasileira de Psicologia (Org.), XXVIII Reunião Anual de Psicologia-Resumos (p. 23). Ribeirão Preto: Autor.

Scarr, S., \& Eisenberg, M. (1993). Child care research: issues, perspectives, and results. Annual Review of Psychology, 44, 613-644.

Scarr, S. (1993). Biological and cultural diversity: the legacy of Darwin for development. Child Development, 64, 1333-1353.

Schaffer, H. R. (1992). Joint involvement episodes as context for development. In H. McGurk (Org.), Childhood social development: Contemporary perspectives (pp.99-129). Hove: Lawrence Erlbaum Associates.

Smith, P. K. (1976). Aims and methods in studying behavioural developmental in children. Ciência e Cultura - Simpósios, 52-62.

Thelen, E. (1989). Self-organization in developmental process: can systems approaches work?. In M. R. Gunnar \& E. Thelen (Org.), Systems and development - The Minnesota Symposia on Child Psychology (vol. 22, pp. 77-117). New York: Lawrence Erlbaum Associates.

Tudge, J., Doucet, F., \& Odero, D. (1999). Desenvolvimento infantil em contexto cultural: o impacto do engajamento de pré-escolares em atividades do cotidiano familiar. Interfaces, 2(1), 23-31.

Tudge, J., Hayes, S., Doucet, F., Odero, D., Kulakova, N., Tammeveski, P., Meltsas, M., \& Lee, S. (2000). Parents' participation in cultural practices with their preschoolers. Psicologia: Teoria e Pesquisa, 16(1), 1-10.

Valsiner, J. (1987). Culture and the development of children's action. A cultural-historical theory of developmental psychology. Chichester: John Wiley \& Sons.

Weisner, T. S., \& Gallimore, R. (1977). My brother keeper: child and sibling caretaking. Current Anthropology, 18, 169-190.

Whiting, B. B. (1983). The genesis of prosocial behavior. In D. L. Bridgment (Org.), The nature of prosocial development (pp. 221-242). Orlando: Academic Press.

Eulina da Rocha Lordelo, doutora em Psicologia Experimental pela Universidade de São Paulo, é Professor Adjunto do Departamento de Psicologia e Faculdade de Educação da Universidade Federal da Bahia. Bolsista CNPq.

Endereço para correspondência: Rua Guadalajara, 15, 101, Morro do Gato, 40.140-460, Salvador, Bahia. Email: eulina@ufba.br. 